Florian Trabert, Mara Stuhlfauth-Trabert, Johannes Waßmer (Hg.) Graphisches Erzählen

\title{
Lettre
}



Florian Trabert,

Mara Stuhlfauth-Trabert,

JOHANNES WASSMER (Hg.)

\section{Graphisches Erzählen}

Neue Perspektiven auf Literaturcomics

[transcript] 
Die Durchführung der Tagung und die Drucklegung des Bandes erfolgten mit freundlicher Unterstützung durch den Lehrförderungsfonds der HeinrichHeine-Universität Düsseldorf.

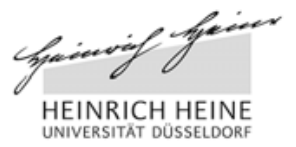

\section{Bibliografische Information der Deutschen Nationalbibliothek}

Die Deutsche Nationalbibliothek verzeichnet diese Publikation in der Deutschen Nationalbibliografie; detaillierte bibliografische Daten sind im Internet über http://dnb.d-nb.de abrufbar.

\section{(ㄷ) 2015 transcript Verlag, Bielefeld}

Die Verwertung der Texte und Bilder ist ohne Zustimmung des Verlages urheberrechtswidrig und strafbar. Das gilt auch für Vervielfältigungen, Übersetzungen, Mikroverfilmungen und für die Verarbeitung mit elektronischen Systemen.

Umschlaggestaltung: Kordula Röckenhaus, Bielefeld;

nach einem Design von Ines Korth, Düsseldorf 2013

Satz: Johannes Waßmer

Redaktionelle Mitarbeit: Robin-M. Aust

Printed in Germany

Print-ISBN 978-3-8376-2825-8

PDF-ISBN 978-3-8394-2825-2

Gedruckt auf alterungsbeständigem Papier mit chlorfrei gebleichtem Zellstoff. Besuchen Sie uns im Internet: $h t t p: / / w w w . t r a n s c r i p t-v e r l a g . d e$

Bitte fordern Sie unser Gesamtverzeichnis und andere Broschüren an unter: info@transcript-verlag.de 